\title{
Estimation and Forecasting of Excitation Force for Arrays of Wave Energy Devices
}

\author{
Yerai Peña-Sanchez, Marina Garcia-Abril, Francesco Paparella and John V. Ringwood, Senior Member, IEEE
}

\begin{abstract}
To maximise energy conversion, real-time control of a Wave Energy Converter (WEC) requires knowledge of the present and future excitation force $\left(F_{\mathrm{ex}}\right)$ acting on the device, which is a non-measurable quantity. The problem of estimation and forecasting of $F_{\mathrm{ex}}$ becomes more challenging when arrays of WECs are considered, due to the hydrodynamic interactions in the array. In this paper, a global $F_{\text {ex }}$ estimator for a complete WEC array is developed and compared to a set of independent estimators which utilise information local only to each device. A significant question is whether the array of measurements is sufficient to compensate for the greater complexity of the wave field, compared to the isolated body case. The paper shows that the global estimator is always more accurate than the independent estimator, improving up to $45 \%$ the estimation accuracy of the independent estimator. Regarding prediction, two different $F_{\text {ex }}$ forecasters for a WEC array are compared: a global forecaster, utilising $F_{\text {ex }}$ estimates from the full set of array devices, and an independent forecaster, utilising only a local $F_{\text {ex }}$ estimate. We demonstrate that the global forecaster achieves more accurate results, not only compared to the independent forecaster, but also compared to the isolated body case.
\end{abstract}

Index Terms-Wave Energy, Excitation Force, Estimation, Forecasting, Wave Energy Converter, Arrays, Autoregressive Model

\section{INTRODUCTION}

A MONG the renewable energy modalities, wave energy has a higher power density than solar or wind energies [1]. In spite of this, the cost involved in generating power from waves is higher than the cost involved in using wind or solar [2]. One way to reduce the electricity generating costs is to maximize the energy extracted from each given Wave Energy Converter (WEC) [3]. Energy maximization can be achieved with an optimal control strategy applied to the WEC [4]. However, an optimal controller requires, in most cases, knowledge of future excitation force $\left(F_{\text {ex }}\right)$ or free surface elevation [4]-[6].

The autoregressive (AR) model has been proven to be a simple but accurate method of predicting both free surface elevation, $\eta$, and $F_{\mathrm{ex}}$ [7] and is adopted here. However, $F_{\text {ex }}$ cannot be directly measured, since there is no absolute reference for a force sensor that can distinguish between the various hydrodynamic forces. One solution is to employ a significant number of pressure sensors over the wetted surface [8], from which $F_{\mathrm{ex}}$ can be deduced, using a suitable model, in a feed-forward manner. Alternatively, $F_{\text {ex }}$ can be estimated using a feedback strategy, based on measurements of the

The authors are with the Department of Electronic Engineering, Centre for Ocean Energy Research, Maynooth University, Maynooth, Co. Kildare, Ireland (e-mail: Yerai.Pena.2017@mumail.ie) position and velocity of a device, which are relatively cheap and straightforward to measure [9] and usually available via the WEC control system.

Commercial WECs are likely to be deployed in arrays, in order to minimise total infrastructural and operational costs [10]. Similarly to the WEC control problem [11], the estimation and forecasting problem for arrays can either be considered from an independent device perspective, where each device has no knowledge of the other devices (distributed or independent control/estimation/forecasting) or from a coordinated, or global, perspective, where the global controller/estimator/forecaster has complete knowledge of the motion of all the devices in the array. Compared to the isolated WEC case (i.e. an 'array' of just a single device), the wave field for multiple devices in an array is significantly more complex, due to the diffraction of the incident waves by each device and the waves which are radiated by each device, by virtue of their motion. A question exists as to whether the increase in information available for the full array is sufficient to counteract this increased wave field complexity, for the estimation and forecasting problems. For the control case, it has been demonstrated that global control always outperforms independent control [11].

The design of an estimator for $F_{\mathrm{ex}}$, based on position and velocity measurements of a single WEC is not new [12]. Regarding prediction, a number of studies have focussed on the prediction of future wave elevation, rather than on the prediction of future $F_{\text {ex }}$ [7]. However, combined estimation/forecasting of $F_{\mathrm{ex}}$ has been addressed [13], [14]. The main novelty in this paper is the consideration of a WEC array, where the wave field is complicated by the presence of diffracted and radiated waves from the array WECs. An estimator, and forecasting model, which has the ability to utilise the full set of WEC device motions available, is designed.

Four different array layouts, as shown in Fig. 1, are examined. In addition, 3 different wave directions $(\beta)$ and 18 different separation distances between devices $(d)$, for each layout, are considered. For each of these cases, two alternative estimation and forecasting models are tested, both global (using all the available measurements) and independent (where the $F_{\text {ex }}$ estimate/forecast for a WEC depends only on the motion information local to that WEC). For the independent estimator, no radiated or diffracted waves from other devices are explicitly taken into account, but rather appear as a supplement to incident (far field) waves. With regard to the forecasting models, the independent model only takes into account past $F_{\mathrm{ex}}$ values of one device to forecast the future 
$F_{\text {ex }}$ acting on that body. However, the global forecasting model uses past $F_{\text {ex }}$ values of all the bodies of the array to forecast $F_{\text {ex }}$.

\section{Layout 1}
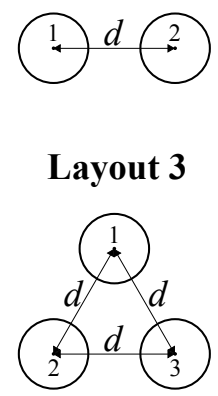

Layout 2

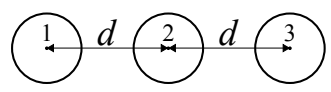

Layout 4

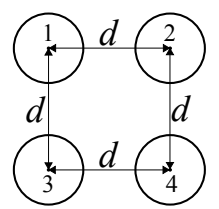

Fig. 1. The different array layouts considered

The remainder of the paper is organised as follows: Section II shows the hydrodynamic model used to describe the WEC array. Section III describes the estimator design, while Section IV presents the forecasting models, which use the estimated $F_{\text {ex }}$ from Section III to predict future $F_{\text {ex }}$ values. Finally, Section $\mathrm{V}$, compares the performances of global and independent estimation and forecasting models, using the isolated WEC case as a reference, and conclusions are drawn in Section VI.

\section{DEVICE MODEL}

The arrays examined comprise a set of cylindrical bodies. All devices are assumed to be identical, with a diameter $(D)$ of $10 \mathrm{~m}$, a draft of $10 \mathrm{~m}$, and a mass $(m)$ of $7.9 e^{5} \mathrm{~kg}$. It is assumed that each device is limited to heave-only motion, and that the WEC array is located in a sea region of relatively small wave amplitude and steepness, so that linear hydrodynamic theory applies. The motion of the bodies of the array is given by Cummin's equation [15],

$$
\left(M+\mathrm{A}_{\infty}\right) \ddot{z}+\int_{t_{0}}^{t} \mathrm{k}_{\mathrm{Rad}}(t-\tau) \dot{z}(\tau) d \tau+K_{H} z+F_{\mathrm{PTO}}=F_{\mathrm{ex}}
$$

where $\mathrm{A}_{\infty}$ is the infinite-frequency added mass asymptote matrix, $K_{H}$ the hydrostatic stiffness matrix, $\mathrm{k}_{\mathrm{Rad}}$ the radiation impulse response matrix, and $z$, $\dot{z}$ and $\ddot{z} n$ length vectors containing the position, velocity and acceleration of the bodies of the array, respectively, where $n$ is the number of bodies of the array. Without loss of generality, we assume that the PTO force $\left(F_{\text {Pто }}\right)$ is zero, since $F_{\text {ex }}$ and $\eta$ are independent of the device motion and the PTO force, for a linear hydrodynamic model.

In Equation (1), the matrices $M$ and $K_{H}$ are $n \times n$ diagonal matrices, containing the values of the mass and the hydrostatic stiffness of each device of the array on the diagonals. Additionally, $\mathrm{A}_{\infty} \in \mathbb{R}^{n \times n}$ is a symmetric matrix, which contains the infinite-frequency added mass asymptote of each body in the diagonal terms and the infinite-frequency added mass asymptote of the interactions in the off-diagonal terms. $A_{\infty}$ matrix is symmetric since, due to the symmetry of the array, the effect of Device $i$ on Device $j$ and vice versa are the same $\left(a_{\infty_{i j}}=a_{\infty_{j i}}\right)$ [16], where $a_{\infty}$ are the components of the matrix $\mathrm{A}_{\infty}$. By way of example, Fig.2 shows the frequency-dependent values of the radiation added mass $\left(A_{R a d}\right)$ for a 3-body array, where the parameters approach their asymptotic $\mathrm{A}_{\infty}$ values on the right-hand side of Fig.2. Finally, $\mathrm{k}_{\mathrm{Rad}}$ contains the radiation impulse response vectors of the individual bodies of the array in the diagonal terms, and the radiation impulse response vector of the interactions in the off-diagonal terms.

In the present paper, the radiation force $\left(F_{\mathrm{Rad}}\right)$ is modelled, as shown in Equation (1), by the convolution integral and $\mathrm{A}_{\infty}$. The radiation impulse response $\mathrm{k}_{\mathrm{Rad}}$ can be obtained, along with $\mathrm{A}_{\infty}$, by using the Ogilvie's relations [17] defined as,

$$
\begin{array}{r}
\mathrm{k}_{\text {Rad }}(t)=\frac{2}{\pi} \int_{0}^{+\infty} \mathrm{B}_{\text {rad }} \cos (\omega t) d \omega \\
\mathrm{A}_{\infty}=\mathrm{A}_{\text {Rad }}+\frac{1}{\omega} \int_{0}^{+\infty} \mathrm{k}_{\text {Rad }}(t) \sin (\omega t) d t
\end{array}
$$

where $\omega$ is angular frequency and both $\mathrm{A}_{\mathrm{Rad}}$ and the radiation damping $\mathrm{B}_{\text {Rad }}$ were obtained using the NEMOH software utility [18]. In the isolated body case, the coefficients of $A_{R a d}$ and $\mathrm{B}_{\mathrm{Rad}}$ are frequency-dependent scalars. However, for the array case, they are two-dimensional matrices of dimension $n \times n$, with frequency-dependent coefficients. At each frequency, the diagonal terms of the $n \times n$ matrices represent the forces measured on a device due to the waves created by its own motion, while the off-diagonal terms represent the coefficients of the radiation forces measured on a device due to the radiated waves created by the motion of other devices, i.e. the interactions.

By way of example, Fig.2 illustrates $A_{\text {Rad }}$ for array Layout 2 , with $d=20 \mathrm{~m}$. Due to the array symmetry, the hydrodynamic coefficients of Devices 1 and 3 are identical $\left(a_{11}=\right.$ $\left.a_{33}\right)$. However, due to its position in the array, the diagonal term corresponding to Device $2\left(a_{22}\right)$ is different. Regarding the non-diagonal terms, due to the symmetry of the array, the waves created from the motion of Device 2 affect Devices 1 and 3 in exactly the same way $\left(a_{12}=a_{21}=a_{23}=a_{32}\right)$. Finally, the effect of Device 1 on Device 3, due to the radiated waves created by the motion of Device 1 , and vice versa, are the same $\left(a_{13}=a_{31}\right)$.

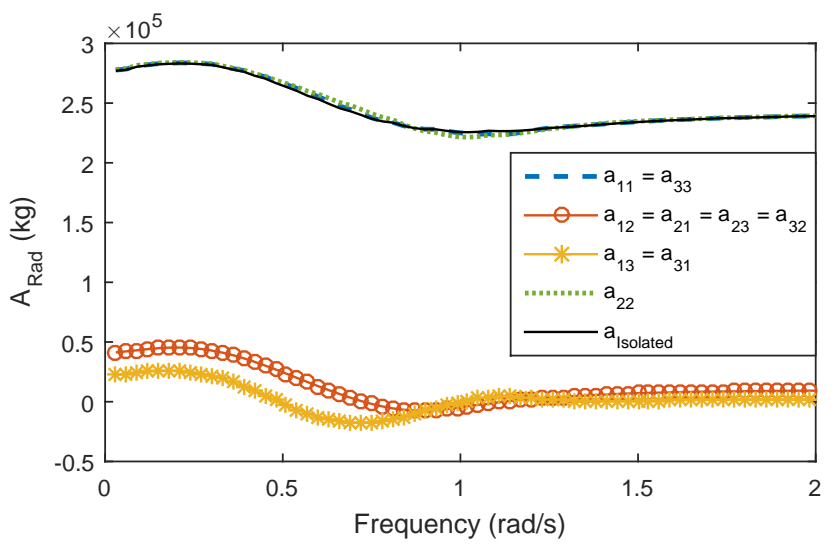

Fig. 2. Radiation added mass of the array Layout 2 and $d=20 \mathrm{~m}$

Fig. 3 shows the radiation damping coefficients of both the bodies and interactions for array Layout 2 with $d=20 \mathrm{~m}$. In 
Fig.3, the same symmetry effects explained for $A_{\text {Rad }}$ in Fig.2 are evident.

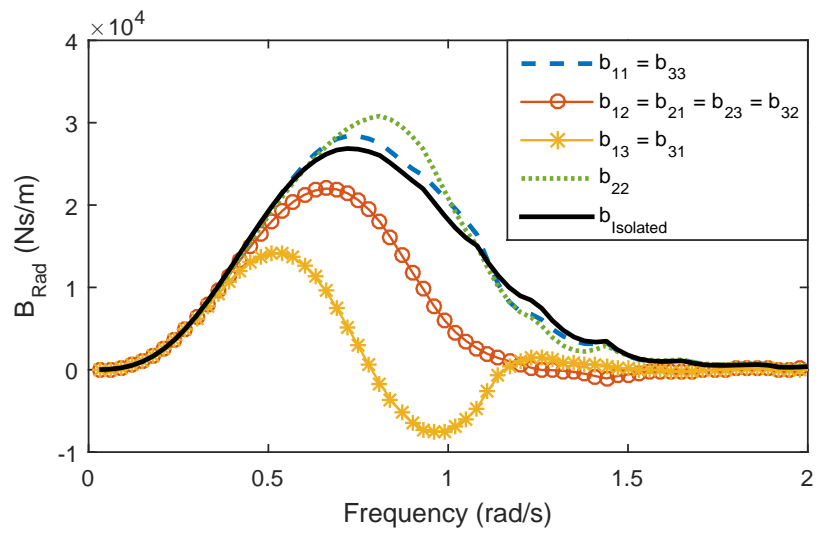

Fig. 3. Radiation damping of the array Layout 2 and $d=20 \mathrm{~m}$

The excitation force $F_{\mathrm{ex}}$ in Eq. (1) is obtained as,

$$
F_{\mathrm{ex}}=\int_{-\infty}^{+\infty} k_{\mathrm{ex}}(t-\tau) \eta(\tau) d \tau
$$

where $k_{\mathrm{ex}}$ is the excitation force kernel, obtained via the inverse Fourier transform of $\mathrm{K}_{\mathrm{ex}}$, supplied via NEMOH. Fig.4 is noteworthy, since it illustrates the variation of $\mathrm{K}_{\mathrm{ex}}$ for the array case, compared to the isolated body case, where $\beta=0^{\circ}$ gives an incident wave in line with the main axis of the array, with waves approaching from the LHS of Fig.1. As a result, the waves diffracted from Device 3 disturb Devices $1\left(\mathrm{~K}_{\mathrm{ex} 1}\right)$ and $2\left(\mathrm{~K}_{\mathrm{ex} 2}\right)$, while the waves diffracted from Device 2 disturb Device $1\left(\mathrm{~K}_{\mathrm{ex} 1}\right)$. Thus, the $\mathrm{K}_{\mathrm{ex}}$ for Device 3 are most similar to the isolated body's $\mathrm{k}_{\mathrm{ex}}$. The small differences between $\mathrm{K}_{\mathrm{ex}}$, for Device $3\left(\mathrm{~K}_{\mathrm{ex} 3}\right)$ and the isolated body, are due to the shadowing effect from the previous devices [19].

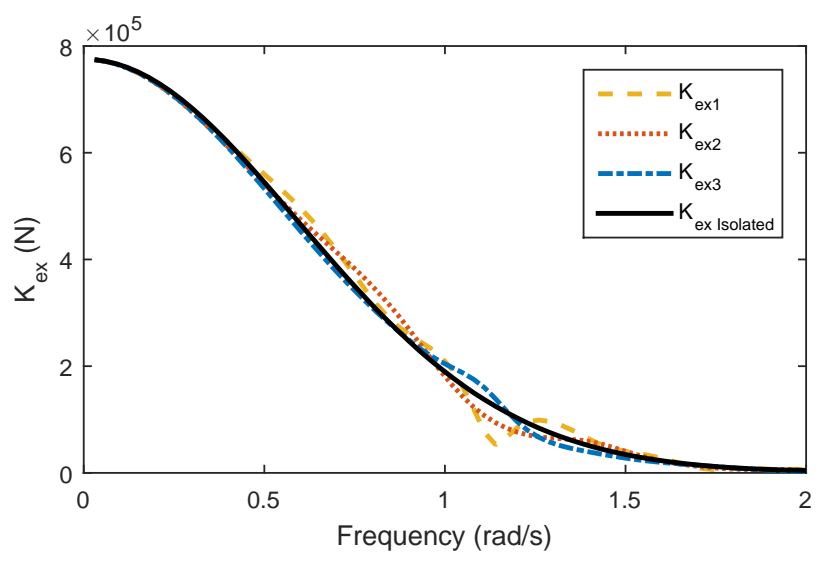

Fig. 4. $\mathrm{K}_{\mathrm{ex}}$ for array Layout $2, d=20 \mathrm{~m}$ and $\beta=0^{\circ}$

The free surface elevation is generated with sets of 200 randomly phased sinusoids to realise the JONSWAP spectrum in each simulation. Furthermore, as detailed in Section V, different random initialisations were performed over multiple simulation runs in order to achieve consistent results. The amplitude of the waves are given by a JONSWAP spectrum of $\gamma=3.3$ with a significant wave height $\left(H_{\mathrm{S}}\right)$ of $1.5 \mathrm{~m}$ and a peak period $\left(T_{\mathrm{P}}\right)$ of $8 \mathrm{~s}$.

The motion of the bodies $(z, \dot{z}$ and $\ddot{z})$ is calculated using second order Runge-Kutta numerical integration. Then, in order to simulate the electrical noise on the real motion measurement sensors, a white noise is added to the measurements of position and velocity of the devices. This white noise's variance corresponds roughly to $1 \%$ of the body displacement.

\section{EXCITATION FORCE ESTIMATION}

In this work, $F_{\mathrm{ex}}$ is estimated based on position and velocity measurements of the devices. A discrete time Kalman Filter $(\mathrm{KF})$ has been developed [20], with a sampling period $\left(T_{\mathrm{s}}\right)$ of $0.01 \mathrm{~s}$. In this work, the dynamical system model is:

$$
\begin{aligned}
\hat{x}_{k} & =A \hat{x}_{k-1}-B \hat{F}_{\text {Rad }_{k-1}}+w_{k-1} \\
y_{k-1} & =H \hat{x}_{k-1}+v_{k-1}
\end{aligned}
$$

where $\hat{x}_{k}$ are the estimated states (position, velocity, excitation force and derivative of the excitation force), $k$ is the discrete time index ( $\left.t=k T_{\mathrm{s}}\right), A$ is the system matrix, $B$ is the input matrix, $\hat{F}_{R_{a d}-1}$ is the estimated value of the radiation force (which is estimated at each step by evaluating the convolution integral in (1) using previous estimated velocity values), $y_{k-1}$ are the measured values of position and velocity, $H$ is the observation matrix, and $w$ and $v$ are, respectively, the process and measurement noise, which are assumed to be stationary and independent, with normal distributions $p(w) \cong N(0, Q)$ and $p(u) \cong N(0, R)$ respectively, where $Q$ and $R$ are the corresponding process covariance matrices. The KF is composed of two parts: a Time Update (TU), in Equation (5), and a Measurement Update (MU), in Equation (6). Time update:

$$
\begin{aligned}
& \hat{x}_{k}^{-}=A \hat{x}_{k-1}-B \hat{F}_{R_{a d}-1} \\
& P_{k}^{-}=A P_{k-1} A^{T}+Q
\end{aligned}
$$

Measurement update:

$$
\begin{aligned}
K_{k} & =P_{k}^{-} H^{T}\left(H P_{k}^{-} H^{T}+R\right)^{-1} \\
P_{k} & =\left(I-K_{k} H\right) P_{k}^{-} \\
\hat{x}_{k} & =\hat{x}_{k}^{-}+K_{k}\left(y_{k}-H \hat{x}_{k}^{-}\right)
\end{aligned}
$$

where $I$ is an identity matrix. In the TU, the states and the error covariance are predicted, i.e. $\hat{x}_{k}^{-}$and $P_{k}^{-}$respectively. In the MU part, the Kalman gain, $K_{k}$, is calculated and the state estimates and the error covariance $\left(\hat{x}_{k}\right.$ and $P_{k}$ respectively) are updated, based on $K_{k}$. In essence, in the TU, the states are estimated and, when new measurements are available, the estimated values are updated in the MU based on the Kalman gain, which indicates the optimal weighting between measured and estimated values. The initial conditions of the $\mathrm{KF}$ are settled to zero.

The estimation precision will depend, mostly, on the accuracy with which the system matrix $(A)$ is defined. The $A$ matrix connects the estimated $F_{\text {ex }}$ with the estimated position and velocity, which are compared with the measured real values at each step. Therefore, the more accurate the relationship between the motion and $F_{\mathrm{ex}}$, the more accurate will be the $F_{\text {ex }}$ estimate. The first $2 n$ rows of $A$, where the motion of the bodies is described, are based on Equation (1). $F_{\mathrm{ex}}$ is described 
in the last $2 n f$ rows of $A$ using a harmonic oscillator model, based on $f$ frequencies, since $F_{\text {ex }}$ is, in general, oscillatory. For further details, refer to [13], [14]. Thus, the system matrix $A \in \mathbb{R}^{(1+f) 2 n \times(1+f) 2 n}$, for an array of $n$ bodies, based on $f$ frequencies, is defined as

$$
A=\left[\begin{array}{cccc}
0_{n, n} & I_{n, n} & 0_{n, n f} & 0_{n, n f} \\
-\mathbf{M}^{-1} K_{H} & 0_{n, n} & \mathbf{M}^{-1} N & 0_{n, n f} \\
0_{n f, n} & 0_{n f, n} & 0_{n f, n f} & I_{n f, n f} \\
0_{n f, n} & 0_{n f, n} & W & 0_{n f, n f}
\end{array}\right]
$$

where $0_{1, n f}$ is a $1 \times n f$ null vector, $I_{n, n}$ a $n \times n$ identity matrix, and $\mathbb{M} \in \mathbb{R}^{n \times n}$ and $N \in \mathbb{R}^{n \times n f}$ are defined as

$$
\begin{gathered}
\mathbb{M}=M+A_{\infty} \\
N=\left[\begin{array}{cccc}
1_{1, n f} & 0_{1, n f} & \cdots & 0_{1, n f} \\
0_{1, n f} & 1_{1, n f} & \cdots & 0_{1, n f} \\
\vdots & \vdots & \ddots & \vdots \\
0_{1, n f} & 0_{1, n f} & \cdots & 1_{1, n f}
\end{array}\right]
\end{gathered}
$$

where $1_{1, n f}$ is a $1 \times n f$ vector of ones, and $W \in \mathbb{R}^{n f \times n f}$ is defined as

$$
W=\left[\begin{array}{cccc}
-I_{n, n}\left(w_{1}\right)^{2} & 0_{n, n} & \cdots & 0_{n, n} \\
0_{n, n} & -I_{n, n}\left(w_{2}\right)^{2} & \cdots & 0_{n, n} \\
\vdots & \vdots & \ddots & \vdots \\
0_{n, n} & 0_{n, n} & \cdots & -I_{n, n}\left(w_{f}\right)^{2}
\end{array}\right]
$$

, while the state vector $x \in \mathbb{R}^{(2+2 f) n \times 1}$ is

$$
x=\left[\begin{array}{lllll}
\hat{x}_{1} \cdots \hat{x}_{n} & \dot{\hat{x}}_{1} \cdots \dot{\hat{x}}_{n} & \hat{F}_{\mathrm{ex}_{11}} \cdots \hat{F}_{\mathrm{ex}_{n f}} & \dot{\hat{F}}_{\mathrm{ex}_{11}} \cdots \dot{\hat{F}}_{\mathrm{ex}_{n f}}
\end{array}\right]_{(11)}^{T}
$$

and $\hat{z}, \dot{\hat{z}}, \hat{F}_{\mathrm{ex}}$ and $\dot{\hat{F}}_{\mathrm{ex}}$ are the estimated values of $z, \dot{z}, F_{\mathrm{ex}}$ and $\dot{F}_{\text {ex }}$, respectively. Matrix $H$ is used to determine which of the states of $\hat{x}_{k}$ can be compared to the measured states $y_{k}$.

$$
H=\left[\begin{array}{ll}
I_{2 n, 2 n} & 0_{2 n, 2 n f}
\end{array}\right]^{T}
$$

As shown in Fig.5, the estimation accuracy of $\hat{F}_{\text {ex }}$ increases with the number of frequencies in the $F_{\text {ex }}$ signal generator. However, $A$ increases by two rows and two columns for each additional frequency, which impacts the computational speed. However, from Fig.5, it is seen that the sensitivity of the estimation accuracy to the number of frequencies is low, beyond 7 frequencies. Therefore, 7 frequencies are used to estimate $F_{\mathrm{ex}}$.

The optimal number of frequencies in $W$ (Equation (10)) and their values depends on the sea state; however, they are not significantly affected by the number of sinusoids used for the simulation, providing the number of simulation frequencies is significantly greater than the number of estimator frequencies. In the current study, 7 frequencies were utilised, with values chosen by using the frequency combination which gives the best estimation accuracy. However, in real life, it is possible to choose the frequencies from a forecasted sea spectrum given by meteorological agencies, and adapt them as the sea state changes.

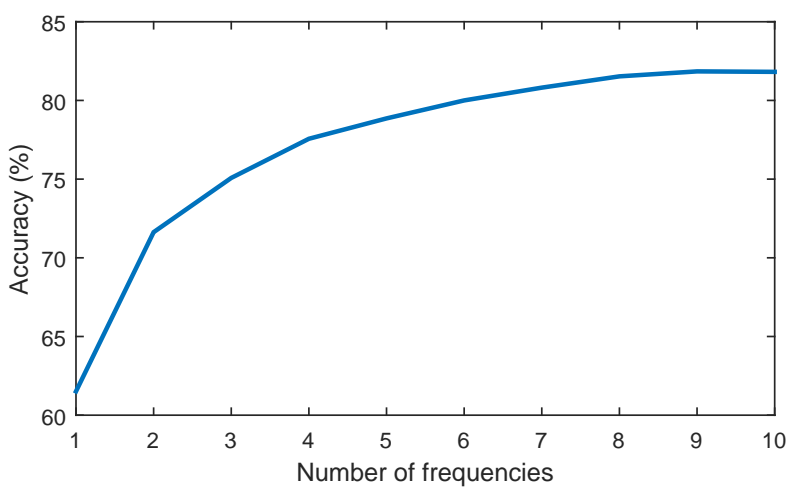

Fig. 5. The accuracy of the estimator for different number of frequency

As shown in Equation (4), the estimated $F_{\text {Rad }}$ is multiplied by the input matrix $B \in \mathbb{R}^{n \times(1+f) 2 n}$, which is defined as:

$$
B=\left[\begin{array}{llll}
0_{n, n} & \mathbb{M}^{-1} & 0_{n, n f} & 0_{n, n f}
\end{array}\right]^{T}
$$

For the independent estimation case an isolated estimator is used in each device. Therefore, since $n=1$, each body works independently, without taking into account any interaction with other bodies of the array. In contrast, the global model takes into account all the interactions with all the bodies of the array. Thus, the size of the $A$ matrix for the global estimator is $n$ times larger than that of the independent estimator. Correspondingly, the computation time for the global estimator increases exponentially with the number of bodies of the array, while the computation time for the independent estimator remains constant.

\section{EXCITATION FORCE FORECASTING}

$F_{\text {ex }}$ is forecasted based on its past estimated values. In particular, in this paper, two different AutoRegressive (AR) models are compared. The first one, the independent forecaster, forecasts one body's $F_{\text {ex }}$ only from a linear combination of past $F_{\text {ex }}$ values of that body. In contrast, the global forecaster uses a linear combination of past $F_{\text {ex }}$ values from all the devices of the array to forecast all the devices' $F_{\text {ex }}$ at the same time. The independent AR model is given as follows:

$$
\bar{F}_{\mathrm{ex}_{k \mid k-1}}=\sum_{i=1}^{h}\left(\phi_{i} \hat{F}_{\mathrm{ex}_{k-i}}\right)
$$

where $\bar{F}_{\mathrm{ex}_{k \mid k-1}}$ is the predicted value of $F_{\text {ex }}$ at instant $k$ from data up to, and including, $k-1, h$ is the order of the forecasting model, $\phi_{i}$ are the autoregressive coefficients. Equation (14) can be written more concisely as

$$
\bar{F}_{\mathrm{ex}_{k \mid k-1}}=\hat{F}_{\mathrm{ex}_{k-1}}^{*} \phi^{*}
$$

where $\hat{F}_{\mathrm{ex}_{k-1}}^{*}$ is a vector of previously estimated $F_{\mathrm{ex}}$ values, as

$$
\hat{F}_{\mathrm{ex}_{k-1}}^{*}=\left[\begin{array}{llll}
\hat{F}_{\mathrm{ex}_{k-1}} & \hat{F}_{\mathrm{ex}_{k-2}} & \ldots & \hat{F}_{\mathrm{ex}_{k-h}}
\end{array}\right]
$$

and $\phi^{*}$ contains the autoregressive coefficients

$$
\phi^{*}=\left[\begin{array}{llll}
\phi_{1} & \phi_{2} & \ldots & \phi_{h}
\end{array}\right]^{T} .
$$


Given a set of training data of dimension $N_{\text {tr }}$, the coefficients of Equation (17) are identified by minimizing the following cost function:

$$
J_{\mathrm{LS}}=\sum_{i=h+1}^{N_{\mathrm{tr}}}\left(\hat{F}_{\mathrm{ex}_{i}}-\bar{F}_{\mathrm{ex}_{i \mid i-1}}\right)^{2}
$$

which is a linear Least Square (LS) problem. Once the $\phi^{*}$ parameters are identified, they are used to predict $F_{\text {ex }}$ values $p$ steps ahead on another data set, where $p=T_{\text {hor }} / T_{\text {sfor }}, T_{\text {hor }}$ is the prediction horizon and $T_{\mathrm{s}_{\text {for }}}$ is the sampling period used for the forecasting. Therefore, the $p$-step-ahead predicted value is obtained as follows:

$$
\bar{F}_{\mathrm{ex}_{k+p \mid k}}=\hat{F}_{\mathrm{ex}_{k+p-1}}^{*} \phi^{*}
$$

where the $p$ step ahead prediction is obtained using an iterative combination of 1-step-ahead predictions.

The global forecasting model used in this paper, which takes into account past $F_{\text {ex }}$ values of all the devices of the array, is:

$$
\bar{F}_{\mathrm{ex}_{b_{k \mid k-1}}}=\sum_{i=1}^{h_{1}} \phi_{b 1_{i}} \hat{F}_{\mathrm{ex}_{b_{k-i}}}+\sum_{\substack{u=1 \\ u \neq b}}^{n} \sum_{j=1}^{h_{2}} \phi_{b u_{j}} \hat{F}_{\mathrm{ex}_{u_{k-j}}}
$$

where $\bar{F}_{\mathrm{ex}_{b_{k \mid k-1}}}$ is the predicted $F_{\mathrm{ex}}$ of Device $b, b=\{1 \ldots n\}$, $\hat{F}_{\mathrm{ex}_{u_{k-j}}}$ the estimated $F_{\mathrm{ex}}$ values of Device $u, h_{1}$ is the order of the model for the body under analysis $(b)$ and $h_{2}$ is the order of the model for the other array bodies. Therefore, as for the independent forecasting model, Equation (20) can be written more concisely as

$$
\bar{F}_{\operatorname{exg}_{k \mid k-1}}=\hat{F}_{\operatorname{exg}_{k-1}^{*}}^{*} \phi_{g}^{*}
$$

where $\bar{F}_{\operatorname{exg}_{k \mid k-1}} \in \mathbb{R}^{n \times 1}$ contains the predicted values of all the bodies of the array, where $\hat{F}_{\operatorname{exg}_{k-1}}^{*} \in \mathbb{R}^{n \times\left(h_{1}+h_{2}(n-1)\right)}$ are the estimated values of $F_{\text {ex }}$ for all devices, as

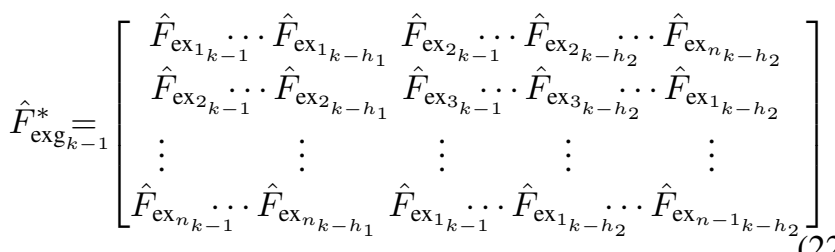

and $\phi_{g}^{*} \in \mathbb{R}^{n \times\left(h_{1}+h_{2}(n-1)\right)}$ are the autoregressive coefficients:

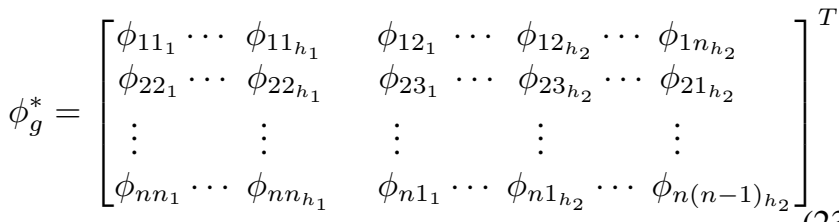

As in the independent predictor model, the coefficients of Equation (23) are identified on a training data (dimension $N_{\text {tr }}$ ), minimizing the following cost function:

$$
J_{\mathrm{LS}}=\sum_{k=r+1}^{N_{\mathrm{tr}}}\left(\hat{F}_{\mathrm{exg}_{k}}-\bar{F}_{\operatorname{exg}_{k \mid k-1}}\right)^{2}
$$

where $r=h_{1}+h_{2}(n-1)$, and $\hat{F}_{\operatorname{exg}_{k}} \in \mathbb{R}^{n \times 1}$ contains the estimated $F_{\mathrm{ex}}$ values for all bodies at instant $k$. As in the independent forecaster, the $p$-step-ahead forecasting is an iterative process. The accuracy of the prediction depends mainly on how many seconds in the past are taken into account to predict a new value which, in turn, depends on the order of the AR and the sampling time. One can notice that a model order of 800 is needed to take into account the last full wave period $(8 s)$, for $T_{\mathrm{s}}=0.01 \mathrm{~s}$. In order to reduce the optimal model order, $\hat{F}_{\text {ex }}$ is re-sampled at $T_{\text {sfor }}$ prior to forecasting.

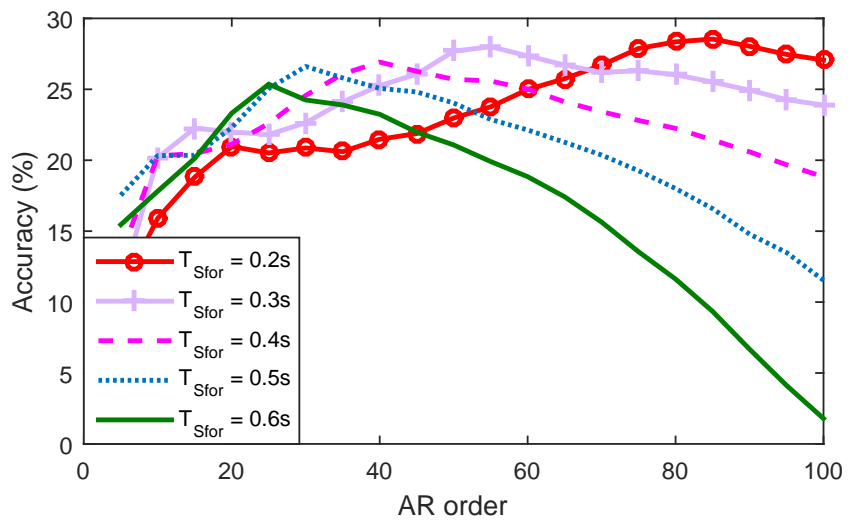

Fig. 6. The accuracy of the prediction using the independent AR with different $T_{\mathrm{s}_{\text {for }}}$ for $T_{\text {horiz }}=4 \mathrm{~s}$

In Fig.6, the forecasting performance, using different $T_{\text {sfor }}$, is illustrated for $T_{\text {horiz }}=4 \mathrm{~s}$. Note that the maximum accuracy for each $T_{\text {sfor }}$ occurs approximately when $16 \mathrm{~s}$ of past values are taken into account, equivalent to two times $T_{\mathrm{P}}$, with $T_{\text {Sfor }}=0.4 \mathrm{~s}$ selected. The resulting optimal order for the independent AR model is then $h=40$. Fig. 6 demonstrates the AR model order necessary to synthesise a frequency response which parsimoniously captures the complexity of the JONSWAP spectrum, with the optimal value of $T_{\text {sfor }}$ related to the ability to appropriately represent the higher wave frequencies. We note that, effectively, the number of discrete frequencies represented by an AR model is half the model order, though this assumes that adjacent frequency amplitudes are not parametrically related, as they are in the JONSWAP model.

Although Fig.6 has been computed with a single body, the used $h$ remains the best order for independent AR in the array case.

Regarding the global predictor, the model order which gives the more accurate prediction varies, depending on the layout, spacing $d$, and wave angle $\beta$. It is attractive to use a consistent order for all cases, with an overall best choice of $h_{1}=40$ and $h_{2}=40$.

The coefficients of the predictors are identified during the first 150s of the simulation $\left(N_{\mathrm{tr}}=375\right)$, which is one third of the total length (450s). The prediction is carried out over the remaining 300s.

\section{RESULTS}

A Goodness of Fit (GoF) metric is used to describe the accuracy of the predicted values, defined as:

$$
\mathrm{GoF}_{f-\bar{f}}=\left(1-\frac{\sqrt{\sum_{k=1}^{N}\left(f_{k+p}-\bar{f}_{k+p \mid k}\right)^{2}}}{\sqrt{\sum_{k=1}^{N}\left(f_{k+p}\right)^{2}}}\right) 100
$$


TABLE I

THE $\mathrm{GOF}_{R}$ OF ESTIMATION FOR LAYOUT 1, FOR DIFFERENT DISTANCES AND WAVE DIRECTIONS

\begin{tabular}{|c|c|c|c|c|c|c|c|c|c|c|c|c|c|c|c|}
\hline & & & & & al esti & ator & & & & & Indep & Ident & imator & & \\
\hline & distances & $2 D$ & $3 D$ & $4 D$ & $5 D$ & $6.5 D$ & $21 D$ & $200 D$ & $2 D$ & $3 D$ & $4 D$ & $5 D$ & $6.5 D$ & $21 D$ & $200 D$ \\
\hline & Device 1 & 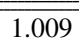 & 1.005 & 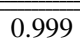 & 1.003 & 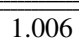 & $\bar{~} 1.004$ & 1.003 & 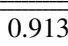 & $\overline{0.963}$ & 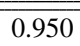 & $\overline{0.964}$ & 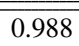 & 0.999 & 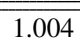 \\
\hline & Device 2 & 1.003 & 1.002 & 1.004 & 1.004 & 1.001 & 1.006 & 1.006 & 0.682 & 0.879 & 0.947 & 0.972 & 0.974 & 1.003 & 1.000 \\
\hline & Device 1 & 1.004 & 1.006 & 0.999 & 0.998 & 1.002 & 1.004 & 1.000 & 0.935 & 0.979 & 0.968 & 0.966 & 0.978 & 0.993 & 1.001 \\
\hline & Device 2 & 1.002 & 1.001 & 1.003 & 1.002 & 1.003 & 1.002 & 1.003 & 0.766 & 0.880 & 0.938 & 0.966 & 0.981 & 0.991 & 1.003 \\
\hline & Device 1 & 1.004 & 1.005 & 1.005 & 1.005 & 1.003 & $\bar{~} 1.002$ & 1.002 & 0.854 & 0.937 & 0.961 & 0.971 & 0.976 & 0.997 & 1.003 \\
\hline & Device 2 & 1.004 & 1.005 & 1.006 & 1.005 & 1.003 & 1.001 & 1.003 & 0.854 & 0.938 & 0.962 & 0.972 & 0.976 & 0.996 & 1.004 \\
\hline
\end{tabular}

where $N$ is the number of forecasted values. In order to use the same quality criteria for estimation and forecasting, the GoF shown in Equation (25) is used also to compute the estimation accuracy. However, since for the estimation, the value of $p$ is 0 , the error is computed between the real value of $f_{k}$ and its estimated value, $\hat{f}_{k}$.

As explained in Section II, in order to evaluate the models in different sea states, multiple sets of phases are randomly generated. However, since the differences between layouts have to be analysed, running only one simulation for each case would lead to results which may not be statistically significant. Thus, in order to determine how many simulations must be carried out to obtain a significant GoF value, an estimate of the GoF mean and variance has been computed with 800 different simulations (using the isolated body case), obtaining a mean GoF of 80.86 and a variance of 0.47 .

Taking into account the obtained variance and assuming the central limit theorem, the relationship between the number of simulations and the $95 \%$ confidence interval can be established. It is found that 35 simulation are necessary to obtain a $95 \%$ confidence interval with a half-width of $0.25 \%$ of the mean, which means that, for a given average GoF estimate obtained from 35 simulations, there is a $95 \%$ probability that the actual average GoF lies within $\pm 0.25 \%$ of the estimated average GoF.

In order to show the differences between the global and independent estimation and forecasting performances, a ratio between the achieved GoF for the arrays and the GoF of the isolated body case is computed as

$$
\mathrm{GoF}_{R}=\frac{\mathrm{GoF}_{\text {array }}}{\mathrm{GoF}_{\text {isol }}}
$$

where $\mathrm{GoF}_{\text {isol }}$ is $80.79 \%$ for the estimations, and the forecasting GoF depends on $T_{\text {horiz }}$. Therefore, the goodness of fit ratio $\left(\mathrm{GoF}_{R}\right)$ shown henceforth, states how accurate are the obtained results of the arrays compared to the isolated body's GoF.

In the graphs shown in this section, the distances between devices $(d)$ are shown in an integer multiples of the device diameter, $D=10 \mathrm{~m}$. Additionally, in Fig.7, since the accuracies of the devices for each layout, $d$ and $\beta$ are similar, only two traces are shown instead of one trace per device and model. These two traces illustrate the mean performance for all bodies, for the global and independent estimators.

In Fig.7, the distance between devices is only shown until $d=100 D$, in order to better appreciate the details of the interactions. As the distance between devices increases, the power density of the radiated and diffracted waves decreases, reducing the magnitude of the interactions. Therefore, as shown in Fig.7, the global and independent estimator accuracies converge on that achieved in the isolated body case, i.e. $\mathrm{GoF}_{R} \rightarrow 1$. Fig.7 also shows that, when the number of bodies of the array increases, the magnitude of the interactions also increases, decreasing the performance of the independent estimators. However, the global estimator $\mathrm{GoF}_{R}$ is always close to unity. Therefore, one can deduce that the extra complexity due to interactions among devices is balanced by the provision of the extra information available to the global estimator.
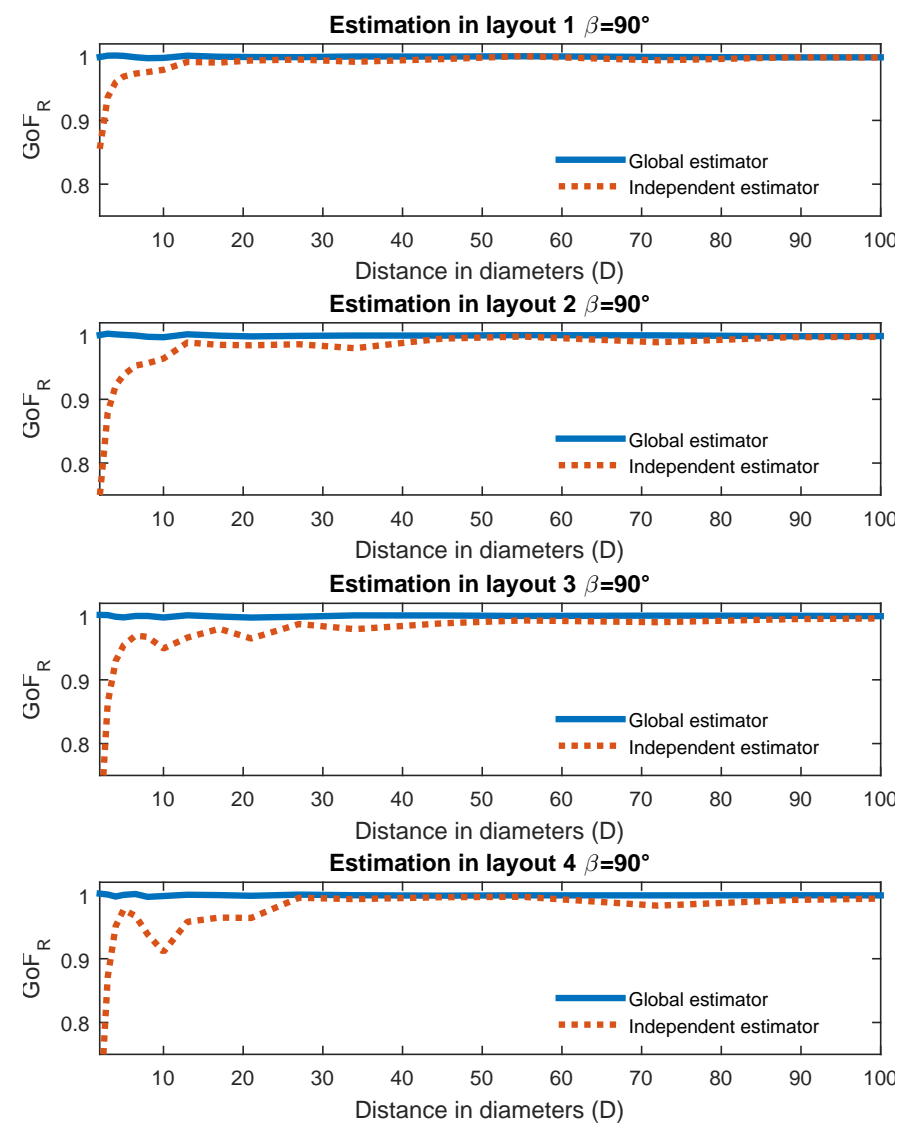

Fig. 7. The estimation $\mathrm{GoF}_{R}$ for different layouts

In Table I, the estimation $\mathrm{GoF}_{R}$ for array Layout 1 is shown for different $\beta$ and $d$. When $\beta=0^{\circ}$ and $\beta=45^{\circ}$, the 
two bodies of the array achieve similar $\mathrm{GoF}_{R}$ using global estimation. However, there is a difference between the two independent estimator performances for close spacing $(d<5 D)$. Nevertheless, when $\beta=90^{\circ}$, both bodies achieve a similar $\mathrm{GoF}_{R}$ for all $d$ with global and independent estimators, due to the symmetry of that case ${ }^{1}$. As for Fig.7, Table I demonstrates that the global estimator always achieves a comparable GoF to the isolated body case, while the independent estimator achieves significantly poorer estimates, particularly when $d$ is small.

Fig. 8 illustrates the variation in the prediction performance when the $\hat{F}_{\text {ex }}$ used to identify the forecasting parameters is obtained from the global and independent estimators, illustrated in (a) and (b), respectively. Only the case for Layout 2, with $\beta=0$ and $d=20 \mathrm{~m}$, is shown in Fig.8, but is representative of all the cases. The predictor, which uses $\hat{F}_{\text {ex }}$ from the global estimator, consistently achieves better prediction compared to the use of $\hat{F}_{\text {ex }}$ from the independent estimators. In addition, since incoming waves first impact Device 1 and, later, Devices 2 and 3 , the knowledge of $\hat{F}_{\text {ex }}$ acting on Device 1 leads to an improvement of the prediction accuracy for Devices 2 and 3. The degree of improvement depends on the distance between Device 1 and Devices 2 and 3 i.e. the amount of time advance in information provided. Thus, Device 3 achieves the best prediction performance and the prediction performance of Device 1 is similar to that achieved in the isolated body case. Only one trace is shown for the independent forecaster, representing the three bodies' forecasting GoF mean.
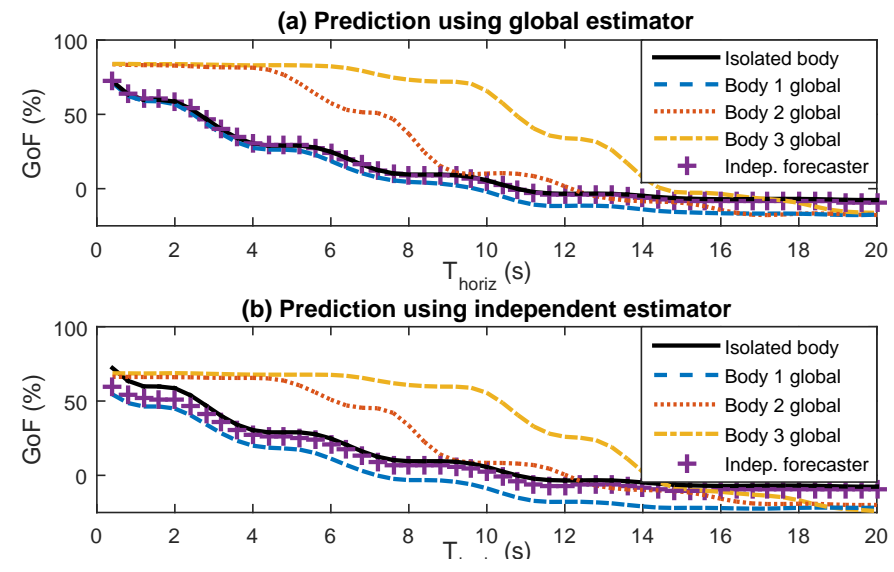

Fig. 8. Comparison of the GoF of the forecasting using the values obtained from the global estimator and independent estimator for Layout 2 with $\beta=0^{\circ}$ and $d=20 \mathrm{~m}$

Fig.9 illustrates the $\mathrm{GoF}_{R}$ of the prediction for different $\beta$, with Layout 2 and $d=20 \mathrm{~m}$. Since the incident wave direction is perpendicular to the main axis of the array for $\beta=90^{\circ}$ (see Fig.9 (c)), all devices are affected by the wave front at the same time. Therefore, there is no greater amount of information from the array motion, compared to the information from any single device. Consequently, the global forecaster achieves a similar GoF to the independent forecaster, and an isolated single body.

\footnotetext{
${ }^{1}$ They are not exactly the same due to the randomness of the noise added to the position and velocity measurements.
}

(a) Prediction using global estimator $\beta=0^{\circ}$

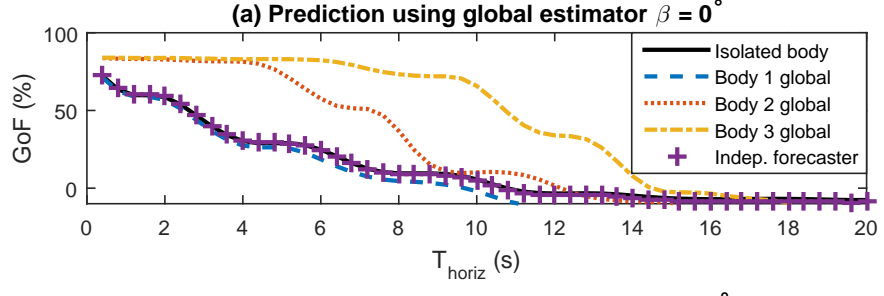

(b) Prediction using global estimator $\beta=45^{\circ}$

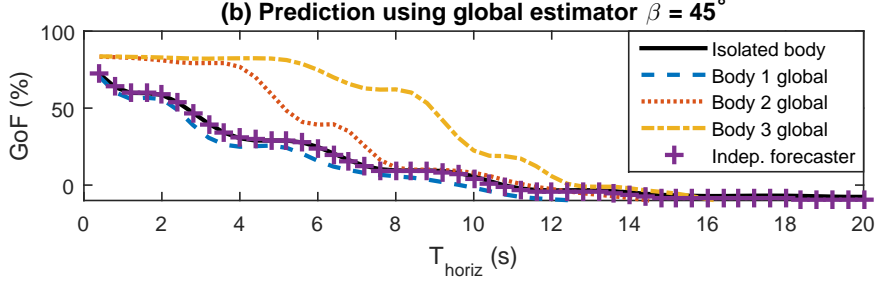

(c) Prediction using global estimator $\beta=90^{\circ}$

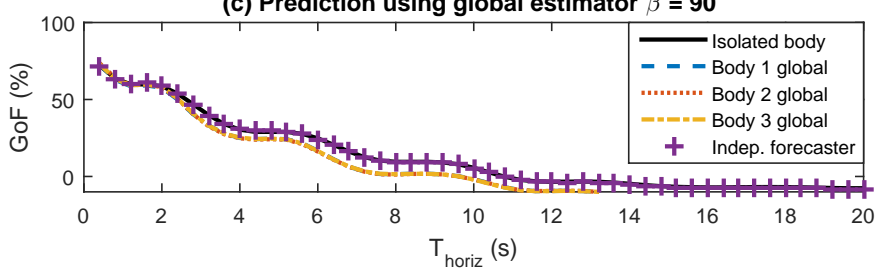

Fig. 9. The GoF of the forecasting using values obtained from the global estimator for Layout 2 with $\beta=0^{\circ}, \beta=45^{\circ}$ and $\beta=90^{\circ}$

Fig.10 again confirms that the estimation of $F_{\text {ex }}$ is more accurate when using the global estimator. Additionally, it is shown that, when using the global predictor, the prediction achieved is quasi-identical to the estimated $F_{\text {ex }}$, due to the anticipative information provided by Devices 3 and 4 .
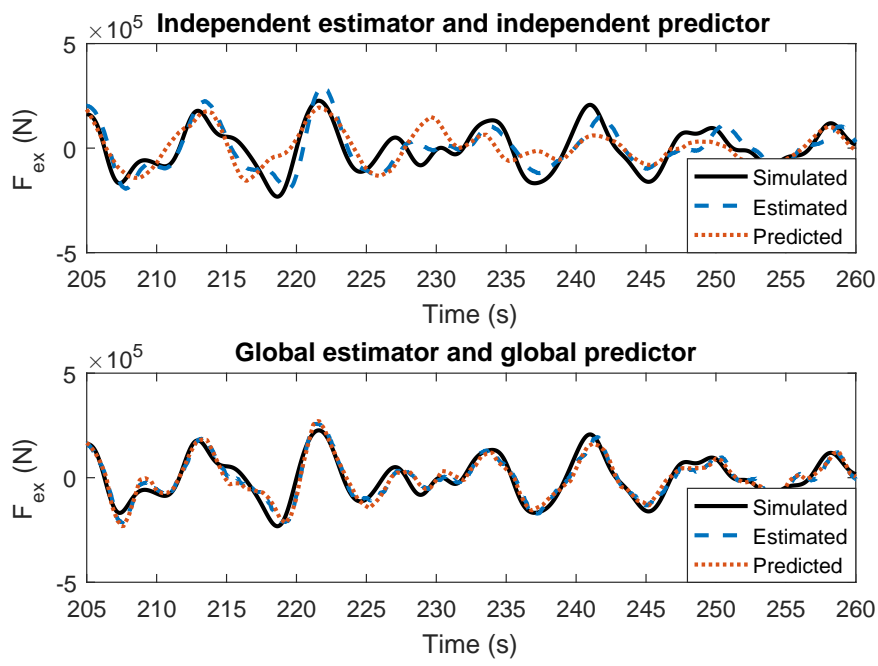

Fig. 10. Section of simulated, the estimated and predicted $F_{\text {ex }}$ using two different combinations of estimator/predictor, for a specific realisation. The device shown is Device 1 of the Layout 4 with $d=20 \mathrm{~m}$ and $\beta=90^{\circ}$. The prediction shown is for $T_{\text {horiz }}=4 \mathrm{~s}$

As explained in Section II, there are cases where the hydrodynamic coefficients are expected to be equal. For example, the excitation force coefficients in Layout 1 , with $\beta=90^{\circ}$, or, as shown in Fig.2, some of the $A_{\text {Rad }}$ coefficients of Layout 3 for any $\beta$ etc. However, in practice, the coefficients obtained from NEMOH, also verified with WAMIT [21], are not exactly 
the same (less than $1 \%$ of variation). Those small differences between the coefficients of the model may be exaggerated during simulation, estimation and forecasting, resulting in a disparity of up to $10 \%$ between the forecasting GoF of devices whose GoF would be expected to be identical, due to symmetry. For this work, that issue is addressed by equalizing the values of the coefficients which must be equal from symmetry considerations.

\section{CONCLUSIONS}

This paper addresses the problem of estimating and forecasting $F_{\text {ex }}$ in an array of WECs which, due to the radiated and diffracted waves, is a more complex problem than for an isolated WEC. In this work, two different $F_{\text {ex }}$ estimators are compared: an independent estimator, which estimates $F_{\mathrm{ex}}$ for each device, based only on motion measurements from the same device, and the global estimator, which estimates $F_{\mathrm{ex}}$ for every device of the array, based on the motion measurements of all array devices. The results show that the complex wave field degrades the independent estimator performance, compared to the global estimator, whose performance is consistent with that of the isolated body case for all the distances, layouts and $\beta$ values analysed.

Regarding forecasting of $F_{\text {ex }}$, two different forecasting models were developed: the independent forecaster, which predicts the $F_{\text {ex }}$ of each device based only on the estimated $F_{\text {ex }}$ from the same device, and the global forecaster, which predicts $F_{\text {ex }}$ for each device based on the estimates of $F_{\text {ex }}$ from all devices. It has been demonstrated that the global forecaster not only achieves better prediction accuracy than the independent forecaster, but also outperforms the isolated body case.

As mentioned in Section IV, the optimal order of the global predictor, which gives the best forecasting accuracy, depends on the layout parameters, $d$ and $\beta$. Thus, slightly better results can be expected from the global forecaster when the order of the AR model is optimally selected for each configuration. However, a pragmatic solution adopts a consistent forecasting model order, for simplicity.

The natural period of the cylinders and the peak period of the sea-state employed in this paper are, respectively, $7.2 \mathrm{~s}$ and $8 \mathrm{~s}$, which suggest that the devices are operating close to resonance. However, higher interactions can be expected when a control strategy is implemented, because the velocity of the devices will increase considerably, with a consequent increase in radiated waves. Therefore, in case of an array under optimal control, there may be a greater disparity between the independent estimator model's performance and that achieved by the global estimator, or isolated body case.

The high computational time needed by the global estimator and forecaster creates some difficulty for real time implementation. Nevertheless, the estimation and forecasting models can be parallelised onto several processors, reducing the sequential computational requirement. In addition, several computational simplifications (e.g. estimator/forecasting model order, etc) could be effected, while retaining the spirit of the global approach.

\section{ACKNOWLEDGEMENTS}

This paper is based upon work supported by Science Foundation Ireland under Grant No. 13/IA/1886.

\section{REFERENCES}

[1] J. Cruz, Ocean wave energy: current status and future perspectives. Springer, 2008.

[2] A. Clement, P. McCullen, A. Falcao, A. Fiorentino, F. Gardner, K. Hammarlund, G. Lemonis, T. Lewis, K. Nielsen, S. Petroncini, M.-T. Pontes, P. Schild, B.-O. Sjostrom, H.-C. Sorenson, and T. Thorpe, "Wave energy in Europe : current status and perspectives," Renewable and Sustainable Energy Reviews, vol. 6, pp. 405-431, 2002.

[3] K. Budal and J. Falnes, "Wave power conversion by point absorbers: A Norwegian project," International Journal of Ambient Energy, vol. 3, no. 2, pp. 59-67, 1982 .

[4] J. V. Ringwood, G. Bacelli, and F. Fusco, "Energy-maximizing control of wave-energy converters: The development of control system technology to optimize their operation," IEEE Control Systems, vol. 34, no. 5, pp. 30-55, 2014.

[5] G. Li, G. Weiss, M. Mueller, S. Townley, and M. R. Belmont, "Wave energy converter control by wave prediction and dynamic programming," Renewable Energy, vol. 48, pp. 392-403, 2012.

[6] G. Bacelli, P. Balitsky, and J. V. Ringwood, "Coordinated Control of Arrays of Wave Energy Devices;Benefits Over Independent Control," Sustainable Energy, IEEE Transactions on, vol. 4, no. 4, pp. 1091-1099, 2013.

[7] F. Fusco and J. Ringwood, "Short-Term Wave Forecasting for Real-Time Control of Wave Energy Converters,' IEEE Transactions on Sustainable Energy, vol. 1, no. 2, pp. 99-106, 2010.

[8] O. Abdelkhalik, S. Zou, G. Bacelli, R. D. Robinett, D. G. Wilson, and R. G. Coe, "Estimation of excitation force on wave energy converters using pressure measurements for feedback control," in OCEANS 2016 MTS/IEEE Monterey. IEEE, 2016.

[9] T. S. Nord, E.-M. Lourens, O. Øiseth, and A. Metrikine, "Model-based force and state estimation in experimental ice-induced vibrations by means of kalman filtering," Cold Regions Science and Technology, vol. 111 , pp. 13-26, 2015

[10] K. Ruehl and D. Bull, "Wave Energy Development Roadmap: Design to commercialization," OCEANS 2012 MTS/IEEE: Harnessing the Power of the Ocean, 2012.

[11] G. Bacelli, P. Balitsky, and J. V. Ringwood, "Coordinated control of arrays of wave energy devices benefits over independent control," IEEE Transactions on Sustainable Energy, vol. 4, no. 4, pp. 1091-1099, 2013.

[12] M. Abdelrahman, R. Patton, B. Guo, and J. Lan, "Estimation of Wave Excitation Force for Wave Energy Converters," Conference on Control and Fault-Tolerant Systems (SysTol), pp. 654-659, 2016.

[13] B. A. Ling and B. A. Batten, "Real time estimation and prediction of wave excitation forces on a heaving body," International Conference on Ocean, Offshore and Arctic Engineering, pp. 1-10, 2015.

[14] M. Garcia-Abril, F. Paparella, and J. Ringwood, "Excitation force estimation and forecasting for wave energy applications," IFAC 2017, 2017.

[15] W. Cummins, "The Impulse Response Function and Ship Motions," 1962.

[16] M. Penalba, I. Touzón, J. Lopez-Mendia, and V. Nava, "A numerical study on the hydrodynamic impact of device slenderness and array size in wave energy farms in realistic wave climates," Ocean Engineering, vol. 142, pp. 224-232, 2017.

[17] T. F. Ogilvie, "Recent progress toward the understanding and prediction of ship motions," in 5th Symposium on naval hydrodynamics, vol. 1, no. 2. Bergen, Norway, 1964, pp. 2-5.

[18] M. Penalba, T. Kelly, and J. V. Ringwood, "Using NEMOH for Modelling Wave Energy Converters : A Comparative Study with WAMIT," 12th European Wave and Tidal Energy Conference, p. 10, 2017.

[19] H. Bernhoff, E. Sjöstedt, and M. Leijon, "Wave energy resources in sheltered sea areas: A case study of the baltic sea," Renewable Energy, vol. 31, no. 13 , pp. $2164-2170,2006$.

[20] F. L. Lewis, L. Xie, and D. Popa, Optimal and Robust Estimation. CRC Press, 2008, vol. 1.

[21] M. WAMIT Inc., WAMIT v7.0 manual, 2013. 


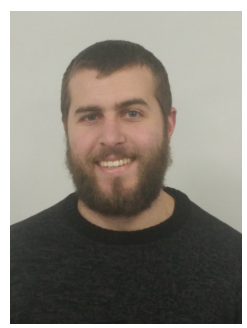

Yerai Peña-Sanchez received the Diploma in Renewable Energies Engineering from the University of the Basque Country (EHU) in 2016. Yerai started his $\mathrm{PhD}$ in October 2016 at the Centre for Ocean Energy Research (COER) in Maynooth University, Ireland. He is working on estimation and forecasting of wave excitation forces.

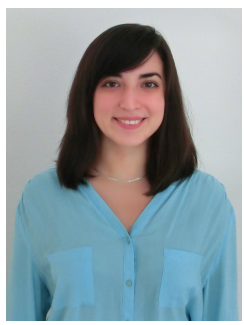

Marina Garcia-Abril finished her BSc in Physics from the University of Valencia in 2015. Then Marina got her MSc in Renewable Energy from the University of Zaragoza in 2016, getting specialised in Ocean Energy from the Instituto Superior Tecnico from Lisbon. She did her internship and final thesis at the Centre for Ocean Energy Research (COER) at NUI Maynooth, modelling an Estimator and forecaster for wave energy applications.

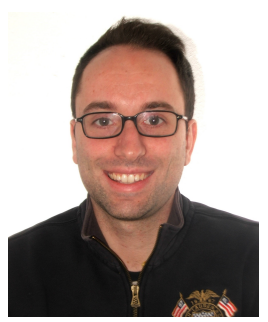

Francesco Paparella received the Laurea Magistrale in Automation Engineering from the Politecnico di Milano in 2012. For his thesis, he worked on the load sharing optimization of compression stations at the ABB Corporate Research Center in Baden (Zurich). He worked from January 2013 up to September 2013 at RSE S.p.a. in Milan as a researcher for the development of electrical systems. Francesco received his $\mathrm{PhD}$ at the Centre for Ocean Energy Research at Maynooth University, Maynooth, Ireland in 2017. He is currently working at the Institute of Technology Blanchardstown as a researcher in wave energy.

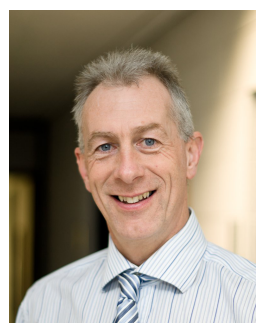

John V. Ringwood received the Diploma in Electrical Engineering from Dublin Institute of Technology and the $\mathrm{PhD}$ (in Control Systems) from Strathclyde University, Scotland in 1981 and 1985 respectively. $\mathrm{He}$ is currently Professor of Electronic Engineering at Maynooth University Ireland and Director of the Centre for Ocean Energy Research there. John's research interests cover time series modelling, wave energy, control of plasma processes and biomedical engineering. He is a Chartered Engineer and a Fellow of Engineers Ireland. 